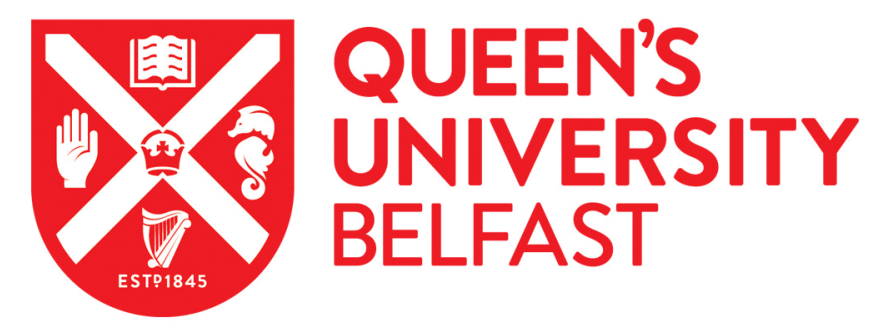

\title{
Theoretical Study of Heteroatom Doping in Tuning the Catalytic Activity of Graphene for Triiodide Reduction
}

Chen, J-F., Mao, Y., Wang, H-F., \& Hu, P. (2016). Theoretical Study of Heteroatom Doping in Tuning the Catalytic Activity of Graphene for Triiodide Reduction. ACS Catalysis, 6(10).

https://doi.org/10.1021/acscatal.6b01242

\section{Published in:}

ACS Catalysis

Document Version:

Peer reviewed version

Queen's University Belfast - Research Portal:

Link to publication record in Queen's University Belfast Research Portal

\section{Publisher rights}

Copyright @ 2016 American Chemical Society

This document is the Accepted Manuscript version of a Published Work that appeared in final form in ACS Catalysis, copyright $\odot$ American Chemical Society after peer review and technical editing by the publisher. To access the final edited and published work see http://pubs.acs.org/doi/abs/10.1021/acscatal.6b01242

\section{General rights}

Copyright for the publications made accessible via the Queen's University Belfast Research Portal is retained by the author(s) and / or other copyright owners and it is a condition of accessing these publications that users recognise and abide by the legal requirements associated with these rights.

Take down policy

The Research Portal is Queen's institutional repository that provides access to Queen's research output. Every effort has been made to ensure that content in the Research Portal does not infringe any person's rights, or applicable UK laws. If you discover content in the Research Portal that you believe breaches copyright or violates any law, please contact openaccess@qub.ac.uk. 


\title{
Theoretical study of heteroatom doping in tuning the catalytic activity of graphene for triiodide reduction
}

\author{
Jian-Fu Chen ${ }^{1}$, Yu Mao ${ }^{2}$, Hai-Feng Wang ${ }^{1}$, and $\mathrm{P} . \mathrm{Hu}^{1,2} *$ \\ ${ }^{1}$ Key Laboratory for Advanced Materials, Research Institute of Industrial Catalysis and Centre for \\ Computational Chemistry, East China University of Science and Technology, Shanghai, 200237, China. \\ Email: hfwang@ecust.edu.cn \\ ${ }^{2}$ School of Chemistry and Chemical Engineering, The Queen's University of Belfast, Belfast, BT9 5AG, UK. \\ Email: p.hu@qub.ac.uk
}

\begin{abstract}
Graphene with heteroatom doping has found increasing applications in a broad range of catalytic reactions. However, the doping effects accounting for the enhanced catalytic activity still remains elusive. In this work, taking the triiodide electroreduction reaction as an example, we study systematically the intrinsic activity of graphene and explore the origin of doping-induced activity variation using first-principles calculations, in which two typical $\mathrm{N}$ and $\mathrm{S}$ dopants are tested. The most common graphene structures, basal plane, armchair edge and zigzag edge, are considered and it is found that the former two structures show a weak adsorption ability for the iodine atom (the key intermediate in the triiodide electroreduction reaction), corresponding to a low catalytic activity. Doping either $\mathrm{N}$ or $\mathrm{S}$ can strengthen the adsorption and thus increase the activity, and the co-doping of N and S (NS-G) exhibits a synergistic effect. A detailed investigation into the whole process of triiodide electroreduction reaction at the $\mathrm{CH}_{3} \mathrm{CN} / \mathrm{NS}-\mathrm{G}$ interface is also carried out to verify these activity trends. It is found that the zigzag edges which contain spin electrons show a relatively stronger adsorption strength compared with the basal plane and armchair edge, and initial doping would result in the spin disappearance that evidently weakens the adsorption; with the disappearance of spin, however, further doping can increase the adsorption again, suggesting that the spin electrons may play a preliminary role in affecting the intrinsic activity of graphene. We also analysed extensively the origin of doping-induced adsorption enhancement of graphene in the absence of spin; it can be rationalized from the electronic and geometric factors. Specifically, N doping can result in a more delocalized "electron-donating area" to enhance I adsorption,
\end{abstract}


while $\mathrm{S}$ doping provides a localized structural distortion, which activate the nearest $\mathrm{sp}^{2}-\mathrm{C}$ into coordinatively unsaturated $\mathrm{sp}^{3}-\mathrm{C}$. These results explain well the improved activity of the doping and the synergistic effect of the co-doping. The understandings are generalized to provide insight into the enhanced activity of oxygen reduction reaction on heteroatom doped graphene. This work may be of importance towards the design of high-activity graphene based material.

Keywords: Graphene, heteroatom doping, first-principles calculation, DSCs, triiodine reduction

\section{Introduction}

Graphene, which comprises one monolayer of $\mathrm{sp}^{2}$ carbon atoms with a hexagonal structure, is recognized as the basic building block of various graphitic materials, ${ }^{1-3}$ it is able to form fullerenes (OD) by wrapping, nanotubes (1D) by rolling up and graphite (3D) by stacking. ${ }^{1,4}$ In recent years, considerable interests have been attracted by this material due to its extraordinary electronic, optical, thermal, and mechanical properties. ${ }^{5-9}$ Pristine graphene, however, exhibits usually limited activity for catalytic reactions; it is insufficient to satisfy diverse demands in the catalytic community. Interestingly, electronic properties of graphene can be altered by introducing heteroatoms into its framework, which can significantly enhance its catalytic performance. ${ }^{10-13}$ Heteroatom doped graphene materials, therefore, have become a hot topic in plenty of research areas, such as dye-sensitized solar cells (DSCs), ${ }^{14-18}$ oxygen reduction reaction (ORR) ${ }^{19-21}$ lithium based batteries, ${ }^{22-24}$ and energy storage. ${ }^{25}$ Among all the heteroatoms, nitrogen is the most used element, since nitrogen and carbon are relatively easy to incorporate together. ${ }^{12,17}$ Beyond nitrogen, ${ }^{19,20}$ other elements such as sulfur, ${ }^{26}$ boron, ${ }^{27}$ phosphorus, ${ }^{28}$ and even selenium ${ }^{29}$ also serve as candidates for doping. In addition to single-element doping, co-doping with two different elements may induce synergistic effects; ${ }^{12}$ this strategy has gained some applications in the design of graphene materials. For instance, Ai et al. ${ }^{22}$ prepared N, S co-doped graphene (NS-G) as anode materials for lithium-ion batteries and a metal-free catalyst for ORR, showing that NS-G possesses a superior performance. Recently, Kannan et al. ${ }^{17}$ reported the synergistic effect of NS-G as the 
counter electrode (CE) for DSCs, suggesting that the presence of heteroatoms breaks the charge neutrality and facilitates the adsorption as well as the subsequent reaction of triiodide/iodide redox couple. Luo et al. ${ }^{18}$ obtained the similar results for DSCs in a different redox couple (disulfide/thiolate), which reinforces the exceptional performance of NS-G as a novel CE material. In addition to $\mathrm{N}$ and $\mathrm{S}$, Razmjooei et al. ${ }^{28}$ observed an enhanced ORR activity by means of doping $\mathrm{P}$ in $\mathrm{N}$ and $\mathrm{S}$ doped graphene. $(\mathrm{B}, \mathrm{N})$ co-doped graphene was also found to display a high efficiency as a metal-free electro-catalyst for ORR by Dai and coworkers. $^{30}$

Despite the uncovered high activities of these heteroatom doped graphene experimentally, there are still some puzzles regarding the doping effect. Why can the heteroatom doping increase the catalytic performance of graphene? What is its intrinsic mechanism? Few theoretical studies were carried out to elucidate these issues with a small size cluster model of graphene. Xia, Dai, and their coworkers ${ }^{21,30-32}$ suggested that it is the electronic spin and charge densities that determine the catalytic capacity of materials for ORR. The introduction of $\mathrm{N}$ atoms will change the conduction band of graphene near the Fermi level and generate net positive charges on $\mathrm{C}$ atoms nearby, resulting in high spin or charge densities on the specific catalytic site. Liang et al. ${ }^{33}$ reached a similar conclusion on NS-G; they found that $\mathrm{C}$ atoms around the doped $\mathrm{N}$ show a higher charge density, while the mismatch of the outermost orbitals of $\mathrm{S}$ and $\mathrm{C}$ results in the $\mathrm{S}$ atom being a catalytic centre. Noticeably, the co-doping of $\mathrm{N}$ and $\mathrm{S}$ will boost the maximum spin density. These investigations focused mainly on the local spin and charge density before and after doping based on a small cluster model. Finite size effects were not taken into account and a systematic examination into the doping effects using the periodic model is therefore desirable. Specifically, the complex structural dependence of graphene, such as basal plane, armchair edge and zigzag edge, which were not explicitly studied in the cluster models, of the doping effects remains elusive. It is clear that the origin of the heteroatom doping effect deserves further investigations. In this work, aiming at establishing a more general model we address these issues by virtue of DFT calculations and in-depth electronic structural analyses. 
In conjunction with our previous work on rational screening of $\mathrm{CE}$ materials of DSCs, ${ }^{34-37}$ we chose the triiodide electroreduction reaction (IRR) as a case study to demonstrate our model in detail, considering that $\mathrm{I}_{3}{ }^{-} / \mathrm{I}^{-}$is an important redox couple. Among the current light-to-energy technologies, DSCs are promising and inexpensive alternatives to the traditional silicon based solar cells to convert solar energy, ${ }^{14,38,39}$ in which the design of cost-effective counter electrode (CE) is an important topic. ${ }^{15,17,18,34-36,40,41}$ Typically, the expensive Pt is used as the CE in DSCs owing to its high activity and superb stability in the $\mathrm{I}^{-} / \mathrm{I}_{3}^{-}$electrolyte. Currently, many materials were studied to replace Pt element, including polymers, ${ }^{40,41}$ transition metal sulphide/carbides/nitrides/oxides, ${ }^{34,35,38}$ carbon materials, ${ }^{15,17,18}$ and so forth. Among these candidates, (N, S) co-doped graphene (NS-G) could be a very promising substitution for Pt as a novel CE material owing to its high efficiency and low cost. Focusing on the practical application, we investigated systematically various model structures (basal planes, armchair edges, and zigzag edges) of graphene and explored the effect of $\mathrm{N}-$, S-doping and their co-doping (denoted as N-G, S-G, NS-G, respectively) on catalytic activity of triiodide reduction in DSC, aiming to uncover the structure-activity relationship. Our work may cater to a wide range of researches especially in energy and graphene related areas.

\section{Methods and Models}

All spin-polarized calculations were carried out using the Vienna ab initio simulation package (VASP), ${ }^{42,43}$ in which we employed Perdew-Burke-Ernzerhof ${ }^{44}$ and optB88 functional with the self-consistent van der Waals (vdW) interaction included ${ }^{45,}{ }^{46}$ unless otherwise specified. The project-augmented wave (PAW) method was used to represent the core-valence interaction. ${ }^{47,48}$ For the calculations of total energy, a cut-off energy of $500 \mathrm{eV}$ was set for plane wave basis sets to expand the valence electronic states. All atoms were optimized using the Broyden-Fletcher-Goldfarb-Shanno minimization scheme until the Hellman-Feynman force of each atom was lower than $0.05 \mathrm{eV} / \AA$.

To systematically describe the properties of graphene, three periodic model structures are considered here (Fig. S1), which contains the basal plane, armchair edge and zigzag edge, respectively. For the basal plane model, a $5 \times 3 \sqrt{3} \times 1$ supercell $(12.34 \AA \times 12.83 \AA \times 15.35 \AA)$ 
with 60 atoms of $\mathrm{C}$ was considered; for the armchair edge, the graphene was expanded in $\mathrm{Y}$ direction and the $7 \times 2 \sqrt{3} \times 1$ supercell was set to be $27.15 \AA \times 8.55 \AA \times 15.00 \AA$, with 60 atoms of $\mathrm{C}$ and 8 atoms of $\mathrm{H}$. Similarly, the zigzag edge model structure was also expanded in $\mathrm{Y}$ direction, corresponding to a $4 \sqrt{3} \times 4 \times 1$ supercell of unit cell $(27.53 \AA \times 9.87 \AA \times 15.00 \AA)$ with 64 atoms of $\mathrm{C}$ and 8 atoms of $\mathrm{H}$. All the edge-carbon atoms were saturated with hydrogen atoms. With respect to each configuration of these three structure models, it is worth noting that different sizes have been tested, which give the same result, guaranteeing the reliability of the model. The Monkhorst-Pack k-point sampling of $2 \times 2 \times 1,1 \times 2 \times 1$, and $1 \times 2 \times 1$ were used for basal plane, armchair edge, and zigzag edge models, respectively. It is worth noting that k-point sampling tests have been carried out, which indicated that these settings can give a reasonable result for the structural optimization and the calculation of adsorption energies (see details in Fig. S2), while all the density of state (DOS) calculations were performed with the k-points of $4 \times 4 \times 1$ in this work. Additionally, the lattice expansion as a result of the heteroatom doping was ignored due to the low doping concentration in a large supercell (see test data in Table S1).

The adsorption energy of I atom $\left(\mathrm{E}_{\mathrm{ad}}^{\mathrm{I}}\right)$ is defined as:

$$
\mathrm{E}_{\mathrm{ad}}^{\mathrm{I}}=\mathrm{E}(\mathrm{I} / \text { substrate })-\mathrm{E}(\text { substrate })-1 / 2 \mathrm{E}\left(\mathrm{I}_{2}\right)
$$

where $\mathrm{E}\left(\mathrm{I} /\right.$ substrate), $\mathrm{E}\left(\right.$ substrate), and $\mathrm{E}\left(\mathrm{I}_{2}\right)$ are the energies of I adsorbed on the substrate, the substrate, and $I_{2}$ in the gas phase, respectively. One can see that, the more negative of $E_{a d}{ }^{I}$ is, the more strongly the species binds with the substrate. The transition states (TS) involved in triiodide reduction are determined by a constrained optimization scheme, ${ }^{49,50}$ which are verified until (i) all forces on atoms vanish; and (ii) the total energy reaches maximum along the reaction coordination but minimum with respect to the rest of the degrees of freedom.

\section{Results and Discussion}

\subsection{Activity descriptor in triiodide reduction reaction}

As reported in our previous work, ${ }^{34-37}$ the reaction mechanism of triiodide reduction reaction, $\mathrm{I}_{3}^{-}(\mathrm{sol})+2 \mathrm{e}^{-} \rightarrow 3 \mathrm{I}^{-}(\mathrm{sol})$ occurring on the CE of DSCs, can be described as follows: 
$\mathrm{I}_{3}{ }^{-}(\mathrm{sol}) \leftrightarrow \mathrm{I}_{2}(\mathrm{sol})+\mathrm{I}^{-}(\mathrm{sol})$

$\mathrm{I}_{2}(\mathrm{sol})+2^{*} \rightarrow 2 \mathrm{I}^{*}$

$\mathrm{I}^{*}+\mathrm{e}^{-} \rightarrow \mathrm{I}^{-}(\mathrm{sol})$

where sol indicates the acetonitrile $\left(\mathrm{CH}_{3} \mathrm{CN}\right)$ solution and * stands for the free site on the electrode surface. One can see that it is a relatively complicated solid/liquid interface reaction, and a comprehensive evaluation of the overall catalytic activity on various catalysts is very challenging. Significantly, our previous work ${ }^{35,51}$ proposed that the adsorption energy of I atom $\left(E_{a d}{ }^{I}\right)$ can serve as an activity descriptor for IRR, and it should usually lie between -0.33 to $-1.20 \mathrm{eV}$ for good catalysts with the optimal one located at $\sim-0.50 \mathrm{eV}^{35,36}$. It can be rationalized by the fact that weak adsorption hinders the molecular dissociation of $I_{2}$, while too strong adsorption would limit the desorption of $\mathrm{I}^{*}$ into $\mathrm{I}^{-}(\mathrm{sol})$. This activity descriptor has been successfully applied to screen CE materials in our previous work. ${ }^{34-37,52}$ Therefore, here we employ $E_{a d}^{I}$ to describe the activity of graphene with and without heteroatom doping. As an initial step, the adsorption of I atom on the pristine graphene was examined as a reference and it gives an $\mathrm{E}_{\mathrm{ad}}^{\mathrm{I}}$ of $0.4 \mathrm{eV}$, which is evidently too weak to make it an ideal CE material for DSCs. With the introduction of heteroatoms in graphene, it is reasonable to expect that, if the adsorption strength toward I can be enhanced, the catalytic activity would be increased (this suggestion was proved by kinetic analysis in later discussion). In the following, we made a systematic theoretical study towards the doping effects by focusing on the I atomic adsorption.

\subsection{Graphene models and its adsorption structures}

Starting from the basal-plane configuration, we optimized the structure of pristine graphene $(G)$, nitrogen-doped and sulphur-doped graphene (see Fig. 1a-f). Similar models have been applied previously to describe graphene with and without heteroatom doping. ${ }^{4,20,32,53}$ We can see that the doped $\mathrm{N}$ atom lies in the plane of graphene (Fig. 1(b, e)), whereas doped $\mathrm{S}$ atom tends to protrude (Fig. 1(c, f)). It can be rationalized by the fact that the bond length of $\mathrm{C}-\mathrm{N}$ and $\mathrm{C}-\mathrm{C}$ are quite similar, whereas the $\mathrm{C}-\mathrm{S}$ bond length is usually 
about $25 \%$ longer than that of the $\mathrm{C}-\mathrm{C}$ bond. ${ }^{13,54}$ The bond length of $\mathrm{C}-\mathrm{C}, \mathrm{C}-\mathrm{N}$, and $\mathrm{C}-\mathrm{S}$ are calculated to be $1.42,1.41$, and $1.74 \AA$ in $\mathrm{G}, \mathrm{N}-\mathrm{G}$ and $\mathrm{S}-\mathrm{G}$, respectively. Then, around the $\mathrm{N}$ or $\mathrm{S}$ atom, there exists a series of adsorption sites; considering the lattice symmetry, some neighbouring and representative adsorption sites (sites 1-5 in Fig. 1b for N-G and sites 1-3 in Fig. 1c for S-G) were examined to identity the most stable adsorption structures (see SI-2 for all the adsorption configurations).

Table 1 shows the calculated adsorption energies. On the pristine graphene, we can see that the I atom is quite far away from the surface with the I-C bond distance $\left(d_{\mathrm{I}-\mathrm{C}}\right)$ at $3.86 \AA$ and corresponds to a very weak adsorption $(0.40 \mathrm{eV})$, which is consistent with the experimentally observed low catalytic activity of pristine graphene. ${ }^{12,16} d_{\mathrm{I}-\mathrm{C}}$ in the most stable adsorption structure on $\mathrm{N}-\mathrm{G}$ and S-G, however, are shortened to $3.13 \AA$ and $3.29 \AA$, respectively, corresponding to enhanced binding strengths with $\mathrm{E}_{\mathrm{ad}}{ }^{\mathrm{I}}=-0.23 \mathrm{eV}$ and $-0.09 \mathrm{eV}$, respectively. Interestingly, we notice that on both N-G and S-G, I adsorbs most preferentially on the $\mathrm{C}$ atom next to the heteroatom. Moreover, the effect of $\mathrm{N}$ doping seems to be delocalized, since the adsorption strength for nearby $\mathrm{C}$ atoms decreases gradually as the distance of $\mathrm{C}$ and $\mathrm{N}$ increases, while the effect of $\mathrm{S}$ doping is more localized since the adsorption strength for nearby $\mathrm{C}$ atoms fade rapidly (see Table 1).

To determine whether there is a possible synergetic effect of doping with two different elements on promoting I adsorption, we investigated the N, S co-doped graphene. Firstly, the stable N, S co-doping configurations were tested. By placing $\mathrm{S}$ atom at a series of doping sites near the $\mathrm{N}$ atom, many co-doping configurations were considered to inspect their stabilities (see the doping sites and their energies in SI-3). We can see from Table S3 that the different adsorption configurations possess similar stabilities. Accordingly, we chose four representative co-doping structures to examine the properties of NS-G, in which the numbers of $\mathrm{C}$ atoms between $\mathrm{N}$ and $\mathrm{S}$ are 1, 2, 3, and 4, respectively. As shown in Figure S3, the doping sites of $\mathrm{S}$ are numbered as $1,2_{1}, 3_{2}$, and $4_{1}$, respectively, denoted as NS-G1, NS-G2, NS-G3, and NS-G4, respectively (Fig. 2, a-d). Secondly, $\mathrm{E}_{\mathrm{ad}}^{\mathrm{I}}$ on different sites on NS-G (1-4) were calculated (Tab. 1). From the table, we can see that the adsorption energies of all NS-G 
configurations are increased to $-0.22 \sim-0.56 \mathrm{eV}$ (see SI-4 for the adsorption structures). Two most stable adsorption configurations are illustrated in Figure 2e and 2f, denoted as NS-G1-1 and NS-G2-1, respectively. For NS-G1-1, S is at the next nearest (NN) site of N, and the I atom adsorbs almost vertically upon the $\mathrm{C}$ (linking with both $\mathrm{N}$ and $\mathrm{S}$ ) with a $d_{\mathrm{C}-\mathrm{I}}$ of $2.63 \AA$ (versus $3.47 \AA$ on pristine graphene). For NS-G2-1, the S atom is at the third nearest (NNN) site of $\mathrm{N}$, and the adsorbed I atom preferably sits on the bridge site of two surface $\mathrm{C}$ atoms that are the nearest to $\mathrm{S}$, as shown in Figure 2f. The corresponding adsorption energies of I are increased to be $-0.56 \mathrm{eV}$ and $-0.42 \mathrm{eV}$, respectively. Compared with both $\mathrm{N}-\mathrm{G}$ and $\mathrm{S}-\mathrm{G}$, which give -0.23 and $-0.09 \mathrm{eV}$ of $\mathrm{E}_{\mathrm{ad}}^{\mathrm{I}}$, respectively, it is evident that NS-G possesses a much stronger $\mathrm{E}_{\mathrm{ad}}^{\mathrm{I}}(-0.56 \mathrm{eV})$, revealing a synergistic effect of the N, S co-doping.

It is worth discussing the testing results of three functionals (PBE, PBE+vdW, optB88-vdW) to clarify the functional influence on $\mathrm{E}_{\mathrm{ad}}^{\mathrm{I}}$. As shown in Table 2, all these three functionals give almost identical trends for $\mathrm{E}_{\mathrm{ad}}^{\mathrm{I}}$ on $\mathrm{G}, \mathrm{N}-\mathrm{G}, \mathrm{S}-\mathrm{G}$, and NS-G, which consolidates the synergistic effect of N, S co-doped graphene. The optB88-vdW, which takes self-consistent potential and the van der Waals interaction into consideration, is generally believed to describe the weak adsorption better ${ }^{53,55}$ and was used throughout the paper as stated in the section of Methods. Recently, Kannan et al. ${ }^{17}$ synthesized NS-G as CE in DSCs $\left(\mathrm{I}_{3}{ }^{-} / \mathrm{I}^{-}\right.$as redox couple) and their experimental data (Table 2) agree well with our results; relative to G, N-G and S-G show a higher performance while NS-G exhibit an exceptional efficiency (NS-G > N-G, S-G > G).

\subsection{Kinetic confirmation of high catalytic activity of NS-G}

To confirm the high activity of NS-G in DSCs, a micro-kinetic investigation into the whole IRR process at the $\mathrm{CH}_{3} \mathrm{CN} / \mathrm{NS}-\mathrm{G}$ interface was carried out. To model the realistic $\mathrm{CH}_{3} \mathrm{CN} / \mathrm{NS}-\mathrm{G}$ interface, several layers of $\mathrm{CH}_{3} \mathrm{CN}$ molecules were explicitly introduced on NS-G surface with a density of $0.79 \mathrm{~g} / \mathrm{cm}^{3}$ under extensive structural optimizations (see details in SI and our previous work ${ }^{35,56}$ ). With the inclusion of solvation effect, $\mathrm{E}_{\mathrm{ad}} \mathrm{I}$ of NS-G increases from $-0.56 \mathrm{eV}$ to $-1.06 \mathrm{eV}$, showing a strong promotion for I adsorption at the 
interface. Then, we explored the whole energy profile on the best adsorption site of NS-G1-1 (Fig. 3a), in which the Gibbs free energy change was estimated for every elementary step of IRR process at $\mathrm{U}_{\text {external }}=0 \mathrm{~V}$ and $\mathrm{T}=300 \mathrm{~K}$ (see SI-5 for calculation details). We found that $\mathrm{I}_{2}$ molecule can readily dissociate into two I* atoms without an obvious dissociation barrier, while such a process is difficult to take place on pristine graphene. Subsequently, the adsorbed iodine atom $\left(\mathrm{I}^{*}\right)$ can receive one electron and desorb into solution in the form of $\mathrm{I}^{-}$. It corresponds to the transition state (TS) with a much elongated $d_{\mathrm{C}-\mathrm{I}}$ of $4.10 \AA$ from the initial $3.49 \AA$, and the $\mathrm{I}$ anion in the solution is surrounded by $\mathrm{CH}_{3} \mathrm{CN}$ molecules with $\mathrm{H}$ atoms in methyl pointing to it (Fig. 3a and 3b). In addition, the charge density differences of the TS were calculated and shown in Figure 3c. We can see that the electrons accumulate at I in the TS (Bader: 0.73 e), resulting from the electron depletion at the surface carbon, heteroatom, and adsorbed $\mathrm{CH}_{3} \mathrm{CN}$ molecules. Moreover, there is no orbital overlap between the p-orbital of $\mathrm{I}^{-}$and the p-orbital of the surface $\mathrm{C}$ atom, indicating a typical ionic bond of $\mathrm{C}-\mathrm{I}$.

To evaluate the activity of NS-G and further understand the process, the energy profiles of IRR on two typical surfaces of the commonly used Pt electrode (i.e. flat $\mathrm{Pt}(111)$ and stepped $\operatorname{Pt}(411))^{35,36}$ were also considered for comparison. In Figure 3d, one can see that the Gibbs free energies of all the elementary steps at the $\mathrm{CH}_{3} \mathrm{CN} / \mathrm{NS}-\mathrm{G}$ interface decrease step by step from the initial state to the final state, implying a considerable catalytic activity. Quantitatively, $\mathrm{E}_{\mathrm{ad}}^{\mathrm{I}}(-1.06 \mathrm{eV})$ of NS-G lies between those of $\operatorname{Pt}(411)$ and $\operatorname{Pt}(111)$, and the corresponding desorption barrier of $\mathrm{I}^{*}\left(\mathrm{E}_{\mathrm{a}}^{\mathrm{des}}\right)$ is only $0.28 \mathrm{eV}$, lower than those at the interfaces of $\mathrm{CH}_{3} \mathrm{CN} / \mathrm{Pt}(111)$ and $\mathrm{CH}_{3} \mathrm{CN} / \mathrm{Pt}(411)$ (0.39 eV and $0.63 \mathrm{eV}$, respectively). Considering that $I_{2}$ is able to dissociate readily on all three surfaces, its dissociation could be expected to be fast and in quasi-equilibrium. Thus, it is reasonable to suggest that $\mathrm{E}_{\mathrm{a}}{ }^{\text {des }}$ determines the overall activity of IRR in NS-G. With this suggestion, we estimated the equilibrium exchange current $\left(\mathrm{i}_{0}\right){ }^{37}$ of IRR by means of micro-kinetic analysis to compare their activity trend. Under experimental conditions $\left(T=300 \mathrm{~K}\right.$, the typical concentration of $\mathrm{I}_{2}$ of 0.03 ), the $\mathrm{i}_{0}$ of the IRR is derived as equation (2) (see SI-6 for details): 


$$
i_{0} \approx-\frac{e k_{B} T}{h} \exp \left(-\frac{\Delta \mathrm{E}_{a}^{\text {des }}}{k_{B} T}\right)
$$

It is clear that there is a quantitative correlation between $\left|i_{0}\right|$ and $\Delta \mathrm{E}_{\mathrm{a}}{ }^{\text {des}}$; the smaller the $\mathrm{E}_{\mathrm{a}}{ }^{\text {des }}$, the higher the catalytic activity. Accordingly, it suggests that the kinetic activities of NS-G towards IRR can even be better than that of Pt, being consistent with the experiments conducted by Kannan et al. ${ }^{17}$ and Luo et al. ${ }^{18}$

\subsection{Origin of enhanced $E_{a d}{ }^{I}$ upon doping and the synergetic co-doping effect}

Having obtained the results above, we are now at a position answer why heteroatoms doping can increase the catalytic activity of graphene. Firstly, we analysed the density of states (DOS) of basal-plane graphene with and without (N, S) doping. From Figure 4, we can see that the Fermi energy levels $\left(\mathrm{E}_{f}\right)$ are increased with the $\mathrm{N}$ or $\mathrm{S}$ doping; in particular, the Fermi energy relative to the vacuum energy level is increased to $-3.62 \mathrm{eV}$ for $\mathrm{NG}$ in comparison with $-4.64 \mathrm{eV}$ for $\mathrm{G}$, while it is also enhanced to $-4.37 \mathrm{eV}$ for $\mathrm{SG}$. Thus, it can be expected in the light of energy level that the electron transfer would be more favoured resulting from the doping, leading then to an increase of the adsorption energy. Further, we examined the number of HOMO electrons before and after doping, which accounts for the electron denotation for I bonding. From the projected density of states (PDOS) shown in Figure S6, we can see that the valence band maximum (VBM) and conduction band minimum (CBM) are mainly comprised of $\mathrm{p}_{\mathrm{z}}$ orbital of $\mathrm{C}$ atoms. We calculated the number of HOMO electrons $\left(\mathrm{n}_{\mathrm{HOMO}}\right)$ by integrating the $\mathrm{p}_{\mathrm{z}}$ band of the basal plane from $-0.5 \mathrm{eV}$ to $0 \mathrm{eV}$ (relative to $\mathrm{E}_{f}$ ), and it shows that $\mathrm{n}_{\mathrm{HOMO}}$ increases considerably with $\mathrm{N}$ or $\mathrm{S}$ doped, being from 0.13 to 0.25 for $\mathrm{N}$ and 0.50 for $\mathrm{S}$ doping (see Table 3). Especially for $\mathrm{S}$ doping, it is evident that more localized $\mathrm{p}_{z}$ bands with a higher energy appear around the Fermi-level (Fig. 4). Secondly, the charge density differences (CDD) for I adsorption were analysed to explore the bonding properties. Figures 5(a-e) show the iso-surfaces of the charge density difference of the graphene before and after heteroatoms doping. Comparing N-G and S-G with the pure graphene, we can see that the bonding characteristics of $\mathrm{N}$ are quite similar to that of $\mathrm{C}$, whereas $\mathrm{S}$ exhibits a significant enhancement of the $\mathrm{p}_{z}$ orbitals of $\mathrm{C}$ atoms nearby. Figures $5(\mathrm{f}-\mathrm{h}, \mathrm{k}-\mathrm{m})$ exhibit the charge 
density differences before and after I adsorption. It is noticeable that electrons accumulate at I, while $\mathrm{C}$ atoms on graphene act as electron donors; moreover, there is no evident orbital overlap between the p-orbitals of $\mathrm{I}$ and $\mathrm{C}$ atoms, showing an ionic bond characteristic. Interestingly, as I adsorbs on pure graphene, only a few $\mathrm{C}$ atoms under I provide electrons to facilitate the bonding (Fig. 4f), while much more $\mathrm{C}$ atoms cooperate in this process on $\mathrm{N}-\mathrm{G}$, resulting in more electrons transferred to I. The effect of S doping, nonetheless, is much localized. Similar to that of pure graphene, only several C atoms under I in S-G would donate electrons. However, each $\mathrm{C}$ atom contributes more electrons ( $\sim .05 e$ on average) (originated from $\mathrm{p}_{\mathrm{z}}$ electrons), leading to a stronger bonding (Fig. 5c and 5h). Bader charge analysis shows that $\mathrm{I}^{*}$ possesses the charges of $0.41,0.59$, and $0.48 e$ on $\mathrm{G}, \mathrm{N}-\mathrm{G}$ and $\mathrm{S}-\mathrm{G}$, respectively. This finding on the bonding mechanism could rationalize the adsorption properties of $\mathrm{N}$ - and S-doped graphene we noticed above (Table 1); $\mathrm{E}_{\mathrm{ad}}{ }^{\mathrm{I}}$ on the $\mathrm{C}$ atoms around $\mathrm{N}$ decrease gradually as the $\mathrm{C}-\mathrm{N}$ distance increases, while for the $\mathrm{S}$ doping case $\mathrm{E}_{\mathrm{ad}}{ }^{\mathrm{I}}$ fade rapidly. To further understand the promotion effect of $\mathrm{N}$ and $\mathrm{S}$ doping, we also considered the local density of state (LDOS) at the ortho and para $\mathrm{C}$ positions around the doping site. We can see from Table 3 that $\mathrm{N}$ doping would lead to redistribution of $\mathrm{p}$ electrons; the occupation of $\mathrm{p}_{z}$ orbital increases and $\mathrm{p}_{\mathrm{x}} / \mathrm{p}_{\mathrm{y}}$ decrease (the $\pi$ conjugation degree decreases), which results in the nearest $\mathrm{C}$ atoms around $\mathrm{N}$ contributing more to the VBM. For $\mathrm{N}$ doping, the contribution of one para-position $\mathrm{C}$ around $\mathrm{N}$ site to the overall VBM would be increased to $8.72 \%$ from the original $4.83 \%$ in the clean graphene, embodying higher reactivity for these nearby $\mathrm{C}$ activated by $\mathrm{N}$ doping. For $\mathrm{S}$ doping, the nearest-neighboured $\mathrm{C}$ would be much more activated with the VBM contribution of each C increased to $24.09 \%$ (ortho) and $19.00 \%$ (para), indicating very high reactivity of a single $\mathrm{C}$ atom near $\mathrm{S}$.

In order to understand the results above, we considered the electronegativity; the Pauling electronegativity of $\mathrm{N}(3.04)$ is evidently larger than $\mathrm{C}(2.55),{ }^{54}$ which leads to the delocalized electrons in graphene inclining to flow to the area near $\mathrm{N}$, thus providing more electrons upon bonding with I. On the other hand, the electronegativity of the S (2.58) and C (2.55) is quite close, and thus the promotion on electron donation is negligible. ${ }^{57}$ To verify this finding, the 
Bader charges for doped graphene relative to pristine graphene were further calculated (Fig. S5). We can see that 2.60 electrons accumulate on $\mathrm{N}$ after doping, indicating that electrons indeed tend to flow to $\mathrm{N}$. With respect to $\mathrm{S}$ doping, $\mathrm{S}$ provides a small amount of electrons to the neighbouring $\mathrm{C}$ atoms and the charge transfer after doping is more moderate. Instead, the enhanced activity of S-G could originate from the localized structural distortion near the $\mathrm{S}$ atom. From Figures $1 \mathrm{c}$ and $\mathrm{f}$, one can see that $\mathrm{S}$ protrudes slightly from the graphene plane. Moreover, the delocalized $\pi$ electrons of graphene would partly break near S. Such a structural effect fades rapidly from the doping site.

For the individual $\mathrm{N}$ or $\mathrm{S}$ doping, our calculations have shed light on the mechanism of the enhanced bonding toward I, which is triggered by a delocalized electronic factor and localized structural factor, respectively. Subsequently, we investigated the electronic structure of N, S co-doped graphene (NS-G1 and NS-G2, Fig. 5i-j), and found that synergistic effects come from the cooperation of these two different promotion effects of N and S. In NS-G1, N and $\mathrm{S}$ are in the meta-position of benzene ring unit, and the $\mathrm{C}$ atom directly linking with $\mathrm{N}$ and $\mathrm{S}$ is thus the most preferential site for I adsorption. As shown in Figure 5i, together with the ionic bond interaction induced by $\mathrm{N}$ doping, the localized structure effect of $\mathrm{S}$ plays a major role and the resulting $\mathrm{sp}^{3}-\mathrm{C}$ overlaps with I atom through the p-orbital, forming a p-p covalent bond. On the other hand, for NS-G2 where N and S are in para-position of a benzene ring unit, $\mathrm{N}$ plays a major role by its delocalized effect; there is an apparent electronic density node between the $\mathrm{C}-\mathrm{I}$ bond, indicating an ionic bond with the Bader charge of $0.63 e$ at I atom. In Figure S5d, both the electron accumulation on $\mathrm{N}$ site and depletion on $\mathrm{S}$ site are strengthened compared with the individual $\mathrm{N}$ and S doping (Fig. S5(b-c)), indicating that $\mathrm{N}$ and S doping effect can complementarily enhance each other. Thus, we can divide the effects of heteroatom in graphene into two categories: (i) The introduction of $\mathrm{N}$ will promote the election donation of nearby $\mathrm{C}$ atoms, representing a delocalized electronic effect; and (ii) the introduction of S will lead to a structural distortion in graphene, facilitating the adsorption of I through a localized structural effect. These two effects can enhance each other in the co-existence systems. 


\subsection{Discussion on armchair and zigzag edge of graphene with and without doping}

To systematically study the doping effect on the catalytic activity of graphene, two common edge structures of graphene, armchair and zigzag configurations, were also tested

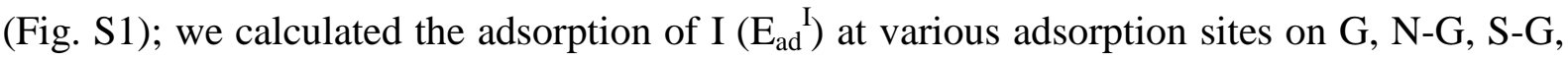
and NS-G, and the most stable ones are summarized in Table 4 (see SI- 8 and SI-9 for all adsorption configurations). One can see that the armchair edge possesses the same trend with the basal-plane structure for the doping effects; N-G and S-G show a higher $\mathrm{E}_{\mathrm{ad}}{ }^{\mathrm{I}}$ than $\mathrm{G}$, and NS-G exhibit a synergetic effect to further enhance $E_{a d}^{I}$. Interestingly, the trend of $E_{a d}{ }^{I}$ on the zigzag edges of graphene, however, displays a different trend. Firstly, the zigzag edge without doping was found to adsorb the I atom strongly $(-0.31 \mathrm{eV})$, which is much stronger than that on the basal phase and the armchair edge $(0.40 \mathrm{eV}$ and $0.35 \mathrm{eV}$, respectively), indicating that the zigzag edge could serve as an active site to promote triiodide reduction. Secondly, with the introduction of doping atoms, the adsorption strength of $\mathrm{I}$ at the $\mathrm{C}$ sites around the dopant becomes weakened on the contrary $(0.12 \mathrm{eV}$ for $\mathrm{N}-\mathrm{G}$; $0.27 \mathrm{eV}$ for $\mathrm{S}-\mathrm{G}$ and $-0.12 \mathrm{eV}$ for NS-G). In other words, the initial $\mathrm{N}$ or S doping does decrease the adsorption ability of zigzag edge, rather than enhance it as do the other two ones.

How can we understand this peculiar phenomenon? In comparison with the nonmagnetic basal plane and armchair edge, the zigzag edges were found to carry unpaired spin electrons mainly distributed along the edge sites with an antiferromagnetic configuration ${ }^{58,59,60}$ (one side of the ribbon is spin-up, while the other side is spin-down) for the tested edge sizes from $6 \times 3$ to $8 \times 8$ (Tab. S4 and Fig. S7). As shown in Figure 6 , these unpaired spin electrons constitute mainly the HOMO orbital. Compared the $\mathrm{p}_{z}$-projected DOS of zigzag edge with the basal-plane configuration, we can see that the zigzag edge possesses a higher Fermi level and a much localized VBM distribution close to the Fermi energy level (Fig. 4), indicating that the zigzag edge has more powerful electron-donation ability and facilitates the binding with I atom. As heteroatoms $\mathrm{N}$ or $\mathrm{S}$ were introduced, the spin electrons of zigzag edge disappeared

(Fig. 6 and Fig. S8), and accordingly $\mathrm{E}_{\mathrm{ad}}^{\mathrm{I}}$ decreased considerably to $\sim 0.2 \mathrm{eV}$. It is worth briefly examining the relationship between the magnetic moment of zigzag edges and the 
corresponding adsorption energy of I. As shown in Figure S10, as the magnetic moment of C site increases, the adsorption energy of I becomes stronger, despite that the correlation becomes poor when the magnetic moment is small. These results suggest that the spin electron plays an important role for the adsorption when the magnetic moment is large enough. Moreover, it is interesting to note that, when doping on one side, the spin electrons remain on the other side of zigzag edge (Fig. 6b) with unchanged HOMO orbital, maintaining its activity unaffected by doping (last column of Table 4). Thus, the presence of unpaired spin electrons appears to play an important role in affecting the adsorption properties of graphene at the zigzag edge. It should be noted that different sized zigzag edge structures are examined on the spin and doping effect (Fig. S7-8 and Tab. S4), and it gives the identical trend.

Figure 6(c-f) show the most favoured adsorption structures of I atom on the doping side of zigzag edge, and the corresponding adsorption energies are listed in the fourth column of Table 4. It shows that the $\mathrm{C}$ directly linked to the $\mathrm{H}$ atom (site 1 , denoted as $\mathrm{C}(1)$ ) possesses the most of spin electrons, and $\mathrm{E}_{\mathrm{ad}} \mathrm{I}$ on this site is $-0.31 \mathrm{eV}$ (Fig. 6c). For $\mathrm{C}(2)$ (site 2), on the other hand, it was unstable for I to adsorb, which will slip back to site 1 upon structure optimization. After doping with $\mathrm{N}$ or $\mathrm{S}$, the spin electrons on the edge decrease drastically; $\mathrm{E}_{\mathrm{ad}}{ }^{\mathrm{I}}$ of N-G and S-G on site 2, which is the nearest the $\mathrm{N}$ or $\mathrm{S}$, were calculated to be 0.12 and $0.27 \mathrm{eV}$, respectively. Nevertheless, as the spins disappear on doping on the zigzag edge, further doping another $\mathrm{N}$ or $\mathrm{S}$ atom would again promote the I adsorption, which is the same as those from the basal-plane structure and armchair edge, supporting the uncovered doping mechanism discovered in this work. For the $\mathrm{N}$ and $\mathrm{S}$ co-doping, $\mathrm{E}_{\mathrm{ad}}{ }^{\mathrm{I}}$ becomes $-0.12 \mathrm{eV}$ on the same doping site (Fig. 6f), much more strengthened than those in N-G and S-G. Compared with the N-doped (or S-doped) zigzag structure without spin, further doping $\mathrm{S}$ (or $\mathrm{N}$ ) to form

$\mathrm{N}$ and $\mathrm{S}$ co-doping would result in an evidently improved $\mathrm{E}_{\mathrm{ad}}{ }^{\mathrm{I}}(-0.12 \mathrm{eV})$, showing similar facilitation as the basal-plane structure and armchair edge.

\subsection{General discussion}

In this section, we address the questions raised in the introduction. Firstly, with detailed 
kinetic analysis of the whole iodine reduction reaction and the inclusion of solvation effects, we demonstrated that the doping of $\mathrm{N}$ or $\mathrm{S}$ in graphene will indeed improve its activity for catalysing triiodide reduction, thus improving the overall performance of the DSSC. Secondly, the co-doping the $\mathrm{N}$ and $\mathrm{S}$, in good agreement with experimental results, shows a synergic effect that increases its activity. Our further analysis displayed that it is the different doping effects, i.e. electronic effect of $\mathrm{N}$ and geometric effect of $\mathrm{S}$, that lead to the synergic effect.

Interestingly, unlike the similar trends of basal plane and armchair edge, the zigzag edge shows a different trend that the adsorption of I will be weaker after the introduction of heteroatoms. Our calculations indicated that there are spin electrons in the zigzag edge, which are in favour of bonding with I atoms. However, after doping with $\mathrm{N}$ or $\mathrm{S}$, the spin electrons disappear and the strength of I adsorption drops significantly. Even though N, S co-doped graphene still shows a stronger $\mathrm{E}_{\mathrm{ad}}^{\mathrm{I}}$ than $\mathrm{N}$ or $\mathrm{S}$ doped graphene, the effect of spin disappearance overwhelms the effect of doping; therefore, the I adsorption strength sequence of them is $\mathrm{G}>\mathrm{NS}-\mathrm{G}>\mathrm{N}-\mathrm{G} \approx \mathrm{S}-\mathrm{G}$.

It is worth noting that the intrinsic mechanism of doping we proposed above can be successfully applied to other graphene-catalysed systems. As mentioned in the introduction, ORR is of significance in energy related areas, in which the heteroatom-doped graphene was verified to give potentially high catalytic activity. Here, we explore the origin of improved performance of ORR resulting from heteroatoms doping using the understanding obtained above. Strong evidences from previous work $^{21,32}$ suggested that $\mathrm{OOH}$ was the key intermediate to evaluate the catalytic activity, and it was revealed that properly increasing the binding energy of $\mathrm{OOH}$ is beneficial for boosting the catalytic activity of graphene-based materials for ORR reaction, according to the obtained volcano-shaped activation trend. ${ }^{61}$ Then we calculated the adsorption energy of $\mathrm{OOH}$ species $\left(\mathrm{E}_{\mathrm{ad}}{ }^{\mathrm{OOH}}\right)$ for $\mathrm{G}, \mathrm{N}-\mathrm{G}$ and $\mathrm{S}-\mathrm{G}$, which are $-0.21,-0.80$ and $-0.72 \mathrm{eV}$, respectively; with respect to the $(\mathrm{N}, \mathrm{S})$ co-doped ones, the $\mathrm{E}_{\mathrm{ad}} \mathrm{OOH}$ were further increased to $-1.89,-0.95$ and $-0.85 \mathrm{eV}$ for NS-G1, NS-G2 and NS-G3, respectively (see SI-11 for all the adsorption configurations). It agrees with the trend we observed in IRR; the adsorption strengthened after doping and N, S co-doping shows a 
synergetic effect. Further, we found that the adsorption energy of $\mathrm{OOH}\left(\mathrm{E}_{\mathrm{ad}}{ }^{\mathrm{OOH}}\right)$ is linearly correlated with $\mathrm{E}_{\mathrm{ad}}^{\mathrm{I}}$ on pure and N/S doped graphene (Fig. S10). This suggests that our uncovered doping mechanism may apply for the case of $\mathrm{OOH}$ adsorption.

\section{Conclusion}

In summary, we have explored the origin of tuned catalytic performance of graphene with heteroatom doping at a molecular level. One important electroreduction reaction, the triiodide reduction, was chosen as a case study. Taking three kinds of graphene models (basal plane, armchair edge, and zigzag edge) into consideration, we systematically examined the adsorption energies of iodine in pristine graphene, $\mathrm{N}$ - and $\mathrm{S}$-doped graphene, as well as the $\mathrm{N}$, S co-doped graphene. The following conclusions are obtained:

(i) On the basal plane and armchair edge, we found that doping with $\mathrm{N}$ or $\mathrm{S}$ increases activity of graphene, and N, S co-doping shows a synergistic effect, enhancing its performance further. This result was verified by a kinetic analysis on the whole iodine reduction reaction with the inclusion of solvation effects.

(ii) After a detailed analysis on the electronic and geometric structure of heteroatom doped graphene, it was found that the effects of heteroatom can be divided into two categories. The effects of first category originate from a difference in electronegativity between doped element and carbon (such as $\mathrm{N}$, generating a delocalized effect by electronic factor), while the second category, from a mismatch of bond length (such as S, generating a localized effect by geometric factor). The synergistic effect of N, S co-doped graphene, consequently, can be well understood by combining electronic and geometric factors together.

(iii) The zigzag edge possesses spin electrons and thus exhibits special properties. Doping one heteroatom would lead to the much decreased adsorption ability rather than the positive effect observed on the basal plane and armchair edge. Analyses of electron structures suggest that the decreased adsorption ability of I on the zigzag edge due to doping one heteroatom results from the disappearance 
of spin electrons and its constituted HOMO orbital on the zigzag edge. However, further doping of $\mathrm{N}$ or $\mathrm{S}$ would again largely facilitate the adsorption, which should obey a similar mechanism uncovered in the basal plane model.

In addition to adsorption of iodine, the explanations of doping effects we proposed above could be generalized to understand the enhanced adsorption strength of $\mathrm{OOH}$ group on heteroatom doped graphene. Heteroatom doped graphene has become a thriving field of materials research and this work may improve our understanding and have some implications towards the design of novel graphene based materials. 

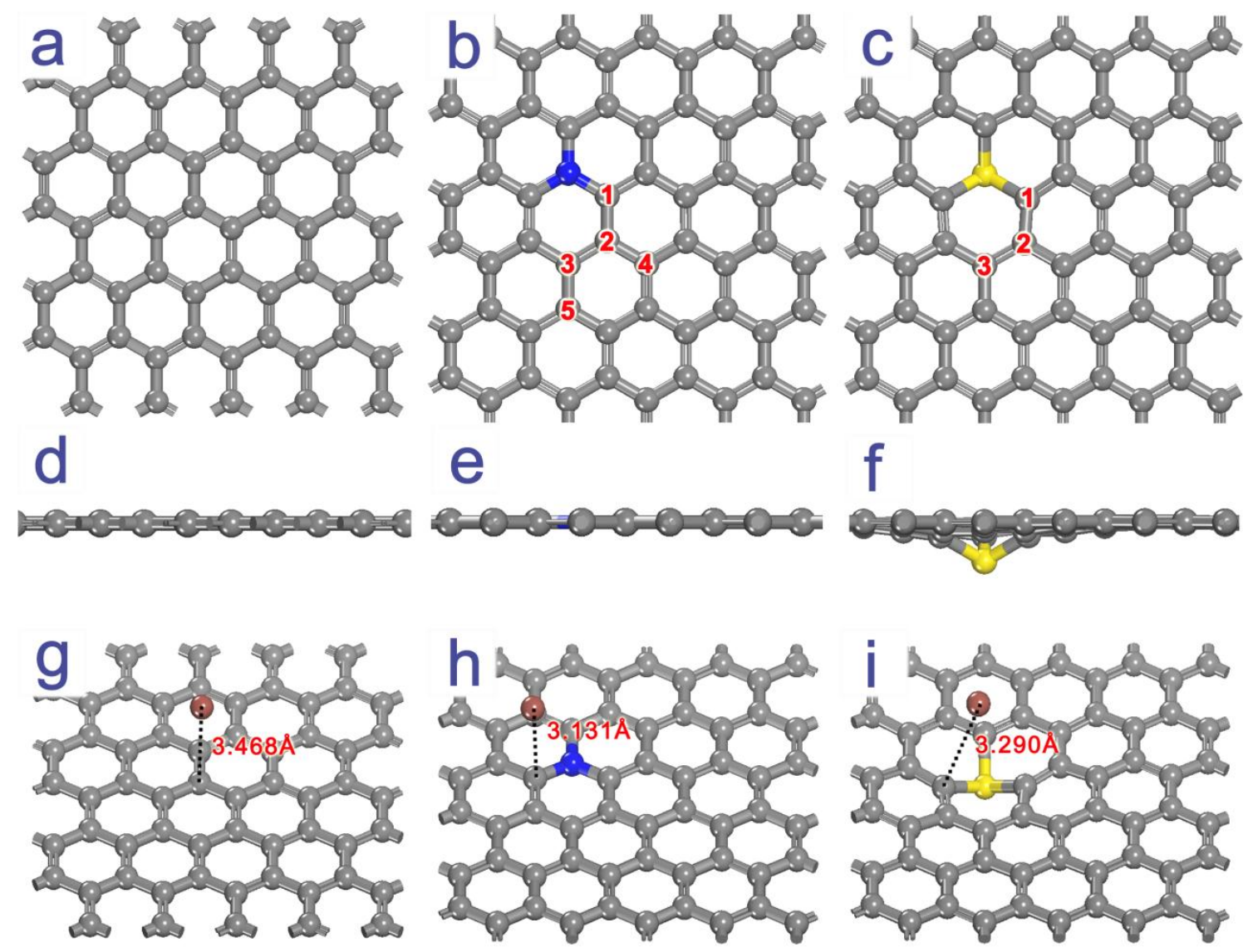

Figure 1. Top and front views of the optimized structures of $G(a, d), N-G(b, e)$, and $S-G(c, f)$ for the periodic basal plane model. (g-i) present the most stable iodine atom adsorption configurations on G, N-G and S-G, respectively, in which the bond lengths of C-I bond were labelled. Numbers in (b) and (c) indicate the selected representative adsorption sites on N-G and S-G. Grey, white, brown, blue, and yellow balls represent C, H, I, N, and S atoms, respectively. This notation is used throughout the paper. 

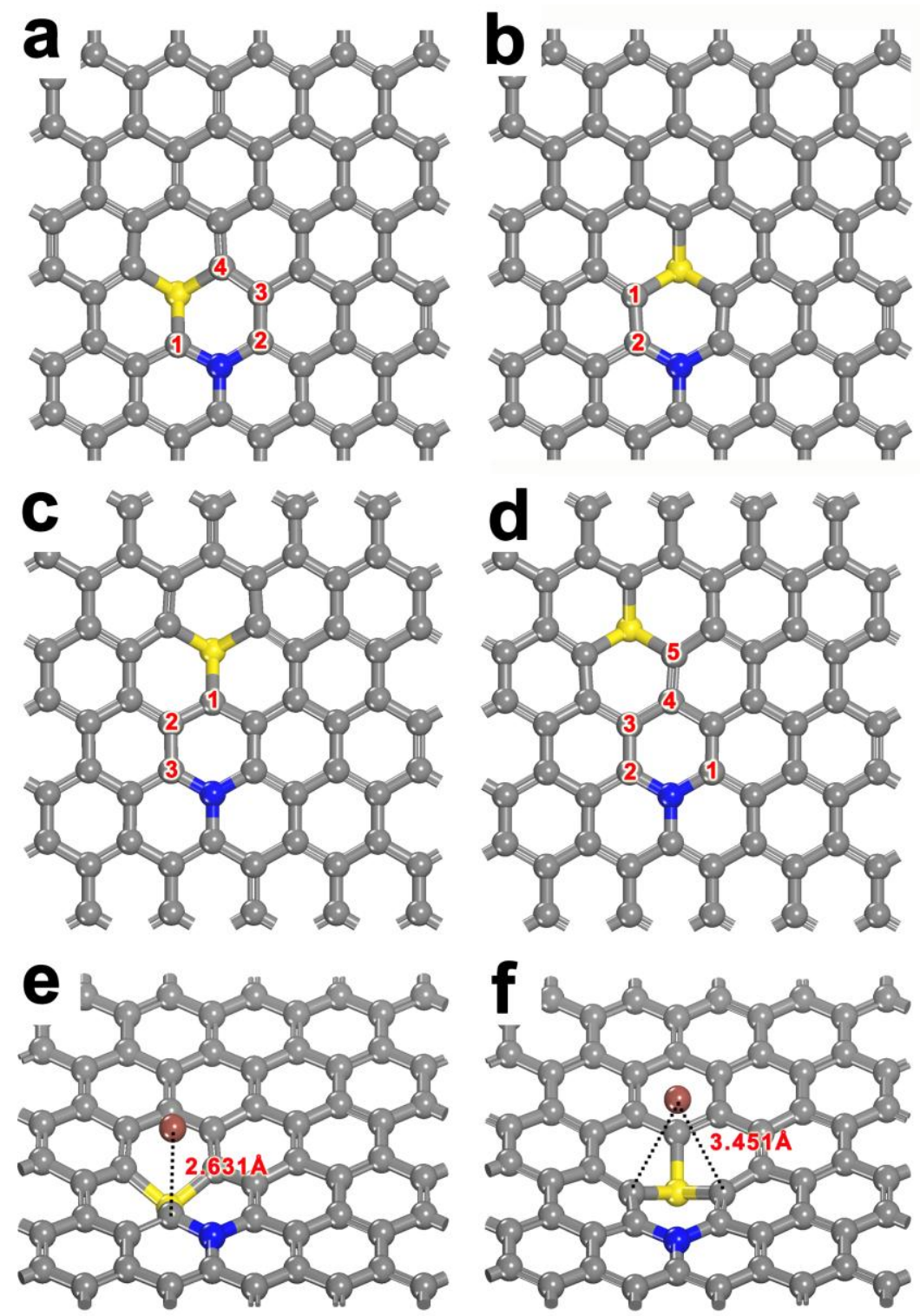

Figure 2. (a-d) illustrate the four $\mathrm{N}$ and $\mathrm{S}$ co-doped graphene structures, denoted as NS-G1, NS-G2, NS-G3 and NS-G4, respectively, on which the possible adsorption sites for I atom were labelled. (e) and (f) show the most stable adsorption configurations on (a) and (b), respectively. 


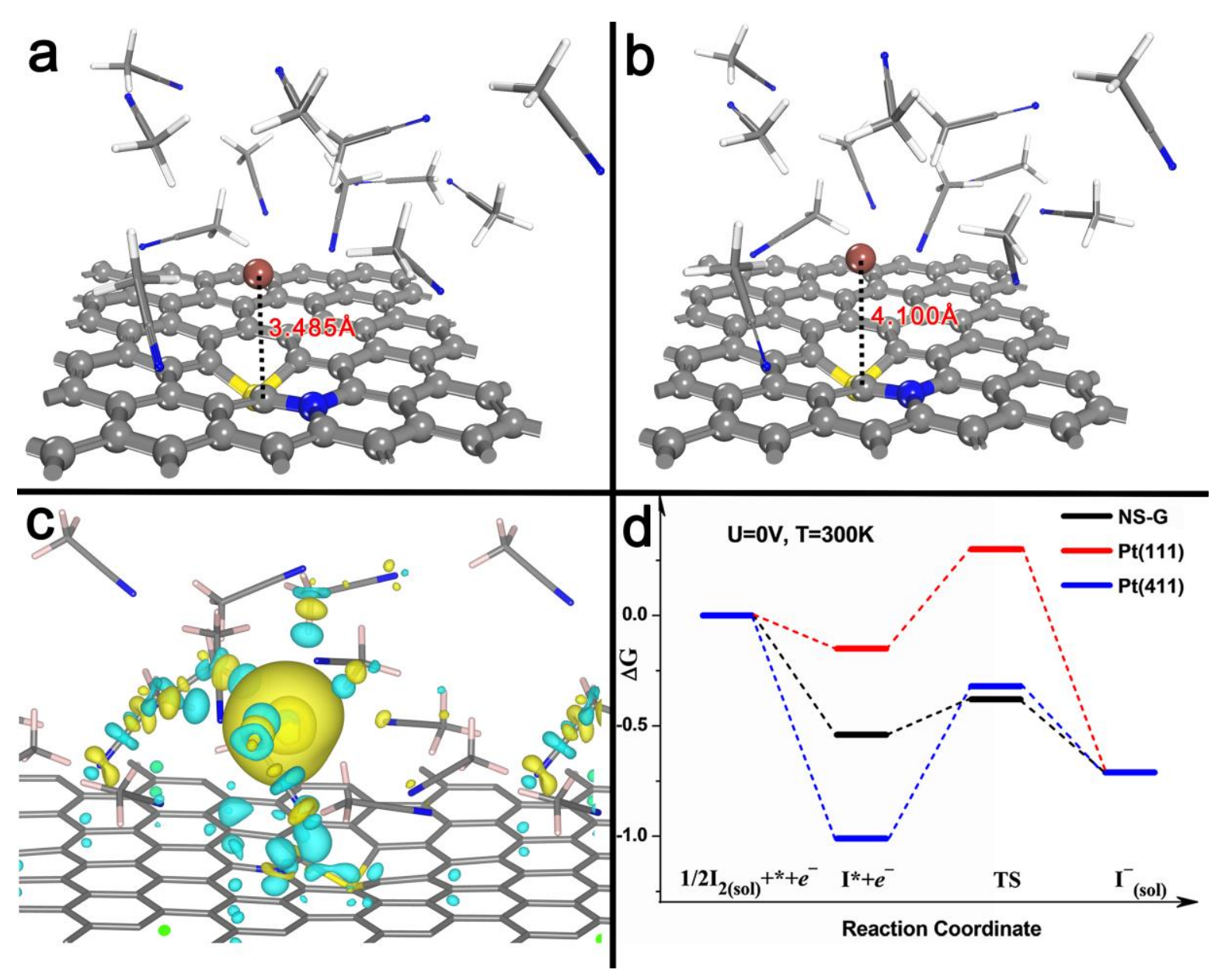

Figure 3. (a) and (b) show the optimized structures of I adsorption and transition state (TS) of I desorption at the $\mathrm{CH}_{3} \mathrm{CN} / \mathrm{NS}-\mathrm{G}$ interface, respectively. (c) illustrates the isosurface of charge density difference for the TS of the I desorption with the value of 0.002 , which is used throughout; yellow indicates the electronic accumulation and light blue for electronic depletion. (d) gives the standard Gibbs free energy profile of IRR at the $\mathrm{CH}_{3} \mathrm{CN} / \mathrm{NS}-\mathrm{G}$ interface, as well as $\mathrm{CH}_{3} \mathrm{CN} / \mathrm{Pt}(111)$ and $\mathrm{CH}_{3} \mathrm{CN} / \mathrm{Pt}(411)$ interfaces. 

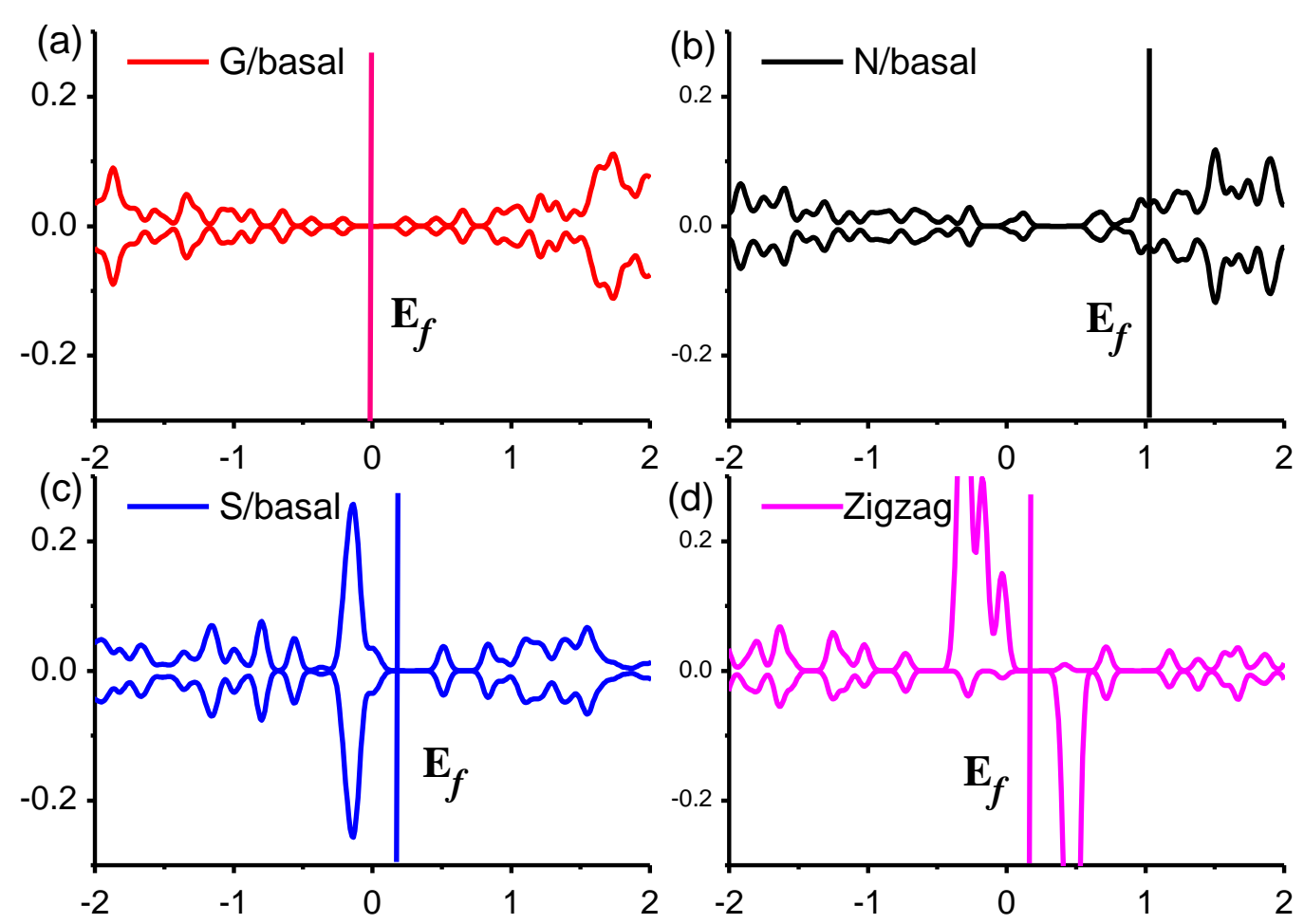

Figure 4. (a-c) The $p_{z}$-projected density of states of the active $C$ atom on the basal plane of graphene before and after $\mathrm{N}$ or $\mathrm{S}$ doping. (b-d) Comparison between the $\mathrm{p}_{\mathrm{z}}$-projected density of states of active $\mathrm{C}$ site on the basal-plane and zigzag-edged. 


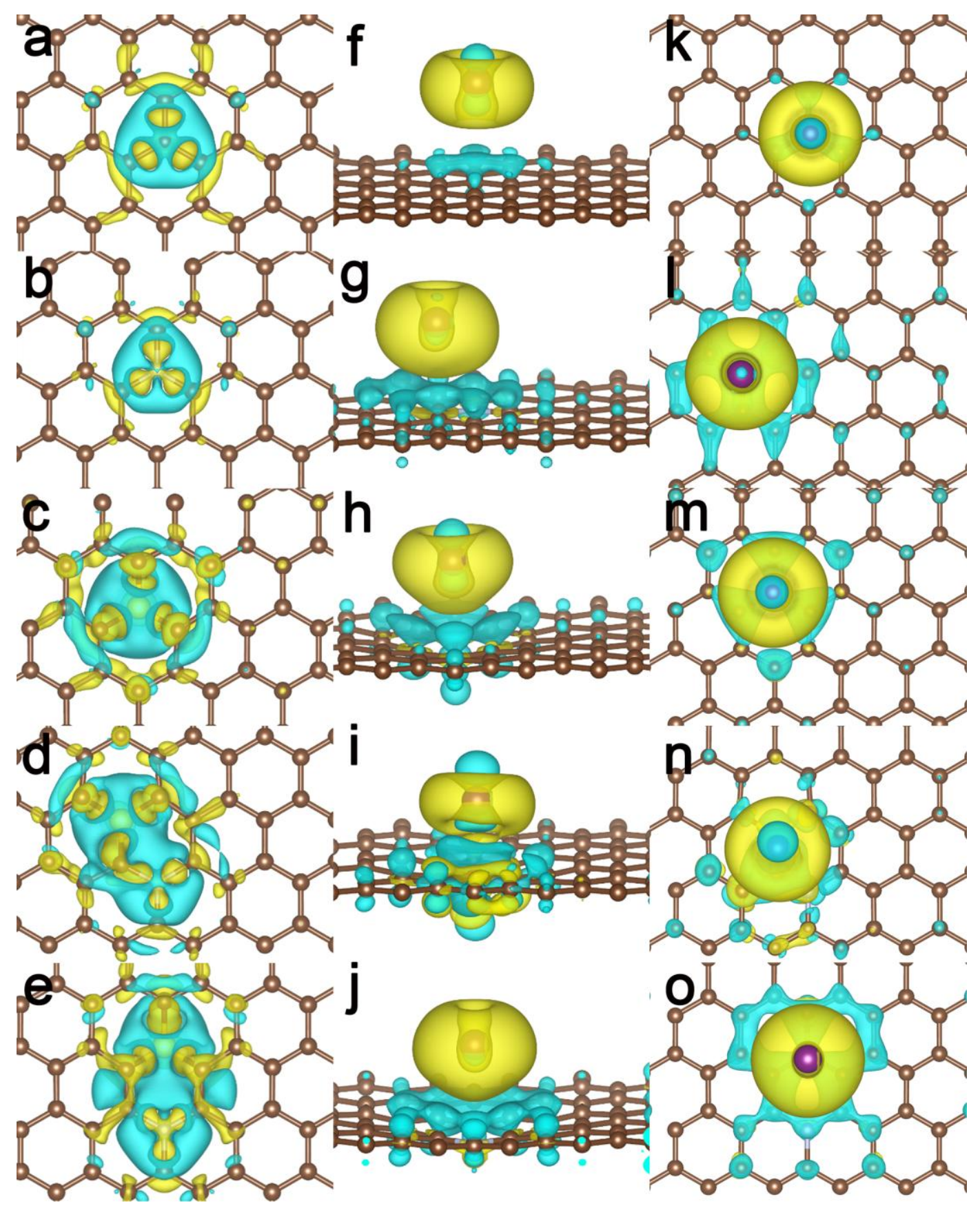

Figure 5. Iso-surfaces of charge density differences. (a-e) are the top views of charge density differences before and after heteroatoms doping on G, N-G, S-G, NS-G1, and NS-G2, respectively. (f-j) are the front views of charge density differences before and after I interacting with $\mathrm{G}, \mathrm{N}-\mathrm{G}, \mathrm{S}-\mathrm{G}, \mathrm{NS}-\mathrm{G} 1$, and NS-G2, respectively, and (k-o) are the corresponding top views. The best adsorption sites for I in (i) and (j), i.e., NS-G1-1 and NS-G2-1, are chosen. 

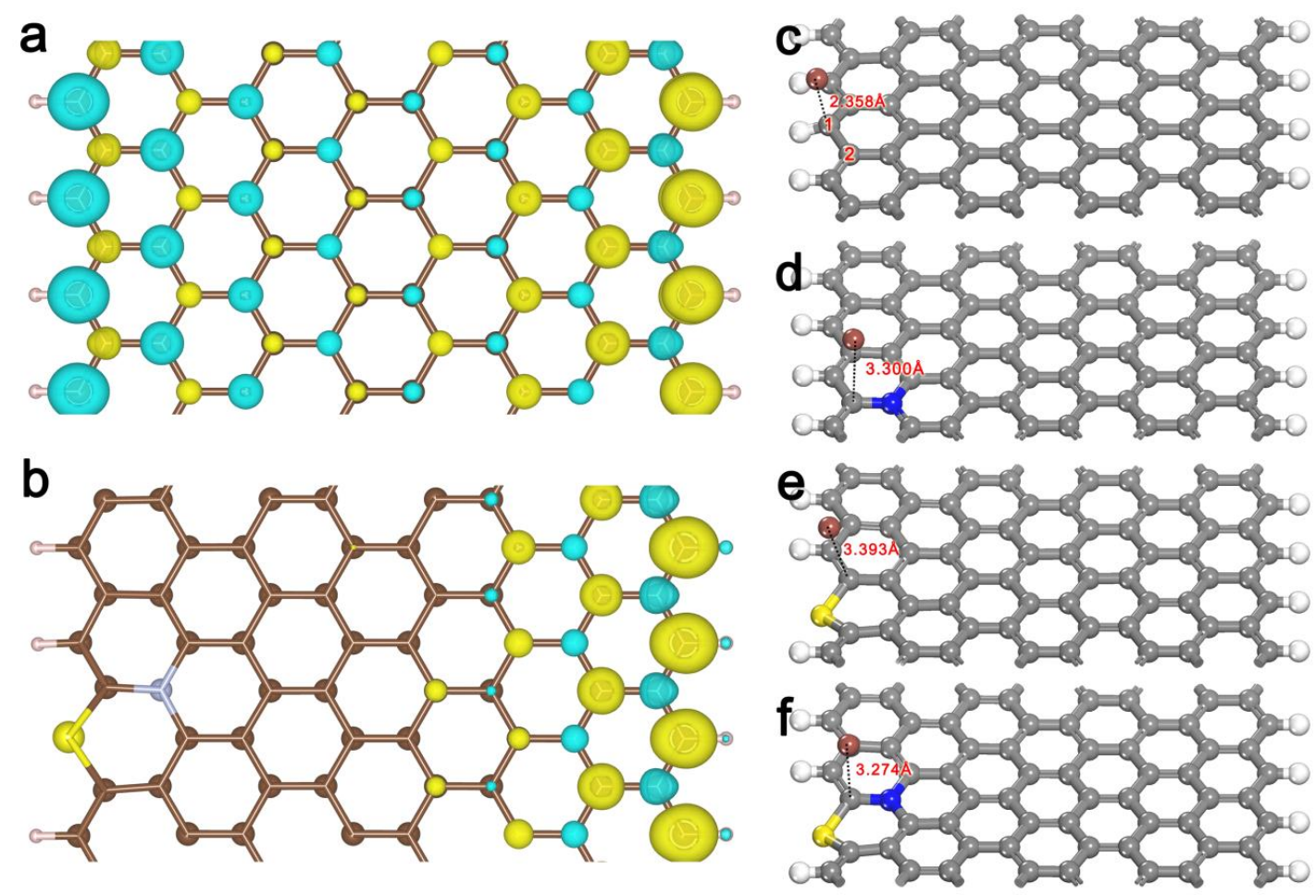

Figure 6. HOMO orbitals of the zigzag edge of graphene before (a) and after (b) N, S co-doping. (c-f) show the most stable adsorption configurations on G, N-G, S-G, and NS-G at the doping side of zigzag edge, and the corresponding adsorption energies are listed in the fourth column of Table 4. 
Table 1. Calculated $\mathrm{E}_{\mathrm{ad}}^{\mathrm{I}}$ on the surfaces of N-G, S-G and NS-G 1-4 with the basal model. Numbers in the first column refer to the corresponding adsorption sites of I on Fig. 1 and Fig. 2

\begin{tabular}{ccccccc}
\hline $\mathbf{E}_{\mathbf{a d}}^{\text {I }} / \mathbf{e V}$ & $\mathbf{N - G}$ & $\mathbf{S - G}$ & NS-G1 & NS-G2 & NS-G3 & NS-G4 \\
\hline $\mathbf{1}$ & -0.23 & -0.09 & -0.56 & -0.42 & -0.38 & -0.36 \\
$\mathbf{2}$ & -0.12 & 0.27 & -0.27 & -0.34 & -0.34 & -0.32 \\
$\mathbf{3}$ & -0.12 & 0.26 & -0.29 & $/$ & -0.34 & -0.27 \\
$\mathbf{4}$ & -0.05 & $/$ & -0.22 & $/$ & $/$ & -0.25 \\
$\mathbf{5}$ & -0.04 & $/$ & $/$ & $/$ & $/$ & -0.35 \\
\hline
\end{tabular}

Table 2. $\mathrm{E}_{\mathrm{ad}}^{\mathrm{I}}$ of the best adsorption structures on surfaces of $\mathrm{G}, \mathrm{N}-\mathrm{G}, \mathrm{S}-\mathrm{G}$, and NS-G. Three different functionals (PBE, PBE+vdW, optB88-vdW) are employed. The last column refers to the efficiency of DSCs synthesized by Kannan et al ${ }^{17}$

\begin{tabular}{ccccc}
\hline $\mathbf{E}_{\mathbf{a d}}^{\mathbf{I}} / \mathbf{e V}$ & PBE & PBE+vdW & optB88-vdW & Experimental $\boldsymbol{\eta} \%$ \\
\hline G & 0.72 & 0.48 & 0.40 & 5.57 \\
N-G & 0.14 & -0.12 & -0.23 & 5.89 \\
S-G & 0.32 & -0.02 & -0.09 & 6.00 \\
NS-G & -0.15 & -0.42 & -0.56 & 7.42 \\
\hline
\end{tabular}


Table 3. Electronic structure properties of basal-plane graphene with and without doping. Columns 2 and 3 are the calculated Fermi levels (E-fermi), number of HOMO electrons ( $\mathrm{n}_{\text {HOмо }}$ ) on the basal plane, respectively, in which $\mathrm{n}_{\text {HOMO }}$ is defined by integrating the $\mathrm{p}_{\mathrm{z}}$ band of the basal plane in the range from $0.5 \mathrm{eV}$ below E-fermi to E-fermi. Columns 5-6 list the numbers of $\mathrm{p}_{\mathrm{z}}$ and $\mathrm{p}_{(\mathrm{x}+\mathrm{y})}$ electrons on the nearest three ortho/para sites around the N/S doping atoms, respectively, while column 7 shows the percentage contribution from these sites to the whole HOMO electron ( $\left.\mathrm{n}_{\text {HOMO }} \%\right)$

\begin{tabular}{lllllll}
\hline & E-fermi & $\mathbf{n}_{(\text {нОмО) }}$ & Sites & $\mathbf{n}_{\mathbf{p z}}$ & $\mathbf{n}_{\mathbf{p}(\mathrm{x}+\mathrm{y})}$ & $\mathbf{n}_{(\text {нОмО) }} \%$ \\
\hline Basal & -4.64 & 0.13 & ortho/para & $1.41 / 1.41$ & $3.94 / 3.94$ & $4.83 / 4.83$ \\
N/Basal & -3.62 & 0.25 & ortho/para & $1.50 / 1.41$ & $3.74 / 3.94$ & $8.72 / 8.72$ \\
S/Basal & -4.37 & 0.50 & ortho/para & $1.65 / 1.51$ & $3.68 / 3.85$ & $24.09 / 19.00$ \\
\hline
\end{tabular}

Table 4. $\mathrm{E}_{\mathrm{ad}}^{\mathrm{I}}$ of the best adsorption structures on the surfaces of $\mathrm{G}, \mathrm{N}-\mathrm{G}, \mathrm{S}-\mathrm{G}$, and NS-G on basal, armchair edge, and zigzag edge. Two sides of zigzag edge are considered (see SI-8 and SI-9 for their structure)

\begin{tabular}{ccccc}
\hline $\mathbf{E}_{\mathbf{a d}} \mathbf{I} / \mathbf{e V}$ & Basal & Armchair & $\begin{array}{c}\text { Zigzag } \\
\text { doping side }\end{array}$ & $\begin{array}{c}\text { Zigzag } \\
\text { non-doping side }\end{array}$ \\
\hline $\mathbf{G}$ & 0.40 & 0.35 & -0.31 & -0.31 \\
N-G & -0.23 & -0.22 & 0.12 & -0.26 \\
S-G & -0.09 & -0.52 & 0.27 & -0.26 \\
NS-G & -0.56 & -1.09 & -0.12 & -0.30 \\
\hline
\end{tabular}




\section{Supporting information}

The Supporting Information is available free of charge on the ACS Publications website:

Details of the computational settings and micro-kinetic analysis, additional electronic and geometrical structures.

\section{Acknowledgements}

This project is supported by National Key Basic Research Program of China (2013CB933201), National Natural Science Foundation of China (21303052, 21333003), Shanghai Rising-Star Program (14QA1401100), “Chen Guang” project (13CG24), Fundamental Research Funds for the Central Universities and Special Program for Applied Research on Super Computation of the NSFC-Guangdong Joint Fund (the second phase). We also thank Prof. Huagui Yang and Dr. Yu Hou for the helpful discussions on the graphene properties.

\section{Reference}

(1) Geim, A. K.; Novoselov, K. S. Nat. Mater. 2007, 6, 183-191.

(2) Randviir, E. P.; Brownson, D. A. C.; Banks, C. E. Mater. Today 2014, 17, 426-432.

(3) Sun, Y.; Wu, Q.; Shi, G. Energ. Environ. Sci. 2011, 4, 1113-1132.

(4) Wang, Z.; Yang, B.; Wang, Y.; Zhao, Y.; Cao, X. M.; Hu, P. Phys. Chem. Chem. Phys. 2013, 15, 9498-9502.

(5) Castro Neto, A. H.; Guinea, F.; Peres, N. M. R.; Novoselov, K. S.; Geim, A. K. Rev. Mod. Phys. 2009, 81, 109-162.

(6) Burgess, R.; Buono, C.; Davies, P. R.; Davies, R. J.; Legge, T.; Lai, A.; Lewis, R.; Morgan, D. J.; Robinson, N.; Willock, D. J. J. Catal. 2015, 323, 10-18.

(7) Nair, R. R.; Blake, P.; Grigorenko, A. N.; Novoselov, K. S.; Booth, T. J.; Stauber, T.; Peres, N. M. R.; Geim, A. K. Science 2008, 320, 1308.

(8) Balandin, A. A.; Ghosh, S.; Bao, W.; Calizo, I.; Teweldebrhan, D.; Miao, F.; Lau, C. N. Nano Lett. 2008, 8, 902-907.

(9) Lee, C.; Wei, X.; Kysar, J. W.; Hone, J. Science 2008, 321, 385-388.

(10) Pumera, M.; Wong, C. H. A. Chem. Soc. Rev. 2013, 42, 5987-5995. 
(11) Kong, X. K.; Chen, C. L.; Chen, Q. W. Chem. Soc. Rev. 2014, 43, 2841-2857.

(12) Paraknowitsch, J. P.; Thomas, A. Energ. Environ. Sci. 2013, 6, 2839-2855.

(13) Wang, X. W.; Sun, G. Z.; Routh, P.; Kim, D. H.; Huang, W.; Chen, P. Chem. Soc. Rev. 2014, 43, 7067-7098.

(14) Hagfeldt, A.; Boschloo, G.; Sun, L.; Kloo, L.; Pettersson, H. Chem. Rev. 2010, 110, 6595-6663.

(15) Hou, S.; Cai, X.; Wu, H.; Yu, X.; Peng, M.; Yan, K.; Zou, D. Energ. Environ. Sci. 2013, 6, 3356-3362.

(16) Xue, Y.; Liu, J.; Chen, H.; Wang, R.; Li, D.; Qu, J.; Dai, L. Angew. Chem. Int. Ed. 2012, 51, 12124-12127.

(17) Kannan, A. G.; Zhao, J.; Jo, S. G.; Kang, Y. S.; Kim, D. W. J. Mater. Chem. A 2014, 2, 12232-12239.

(18) Luo, Q.; Hao, F.; Wang, S.; Shen, H.; Zhao, L.; Li, J.; Grätzel, M.; Lin, H. J. Phys. Chem. C 2014, 118, 17010-17018.

(19) Deng, D.; Yu, L.; Pan, X.; Wang, S.; Chen, X.; Hu, P.; Sun, L.; Bao, X. Chem. Commun. 2011, 47, 10016-10018.

(20) Yu, L.; Pan, X.; Cao, X.; Hu, P.; Bao, X. J. Catal. 2011, 282, 183-190.

(21) Zhang, L.; Niu, J.; Dai, L.; Xia, Z. Langmuir 2012, 28, 7542-7550.

(22) Ai, W.; Luo, Z.; Jiang, J.; Zhu, J.; Du, Z.; Fan, Z.; Xie, L.; Zhang, H.; Huang, W.; Yu, T. Adv. Mater. 2014, 26, 6186-6192.

(23) Shu, C.; Li, B.; Zhang, B.; Su, D. ChemSusChem 2015, 8, 3973-3976.

(24) Ma, X. L.; Ning, G. Q.; Sun, Y. Z.; Pu, Y. J.; Gao, J. S. Carbon 2014, 79, 310-320.

(25) Chen, C. M.; Zhang, Q.; Zhao, X. C.; Zhang, B.; Kong, Q. Q.; Yang, M. G.; Yang, Q. H.; Wang, M. Z.; Yang, Y. G.; Schlögl, R.; Su, D. S. J. Mater. Chem. 2012, 22, 14076-14084.

(26) Lin, T.; Tang, Y.; Wang, Y.; Bi, H.; Liu, Z.; Huang, F.; Xie, X.; Jiang, M. Energ. Environ. Sci. 2013, 6, 1283-1290.

(27) Sheng, Z.-H.; Gao, H.-L.; Bao, W.-J.; Wang, F.-B.; Xia, X.-H. J. Mater. Chem. 2012, 22, 390-395.

(28) Razmjooei, F.; Singh, K. P.; Song, M. Y.; Yu, J. S. Carbon 2014, 78, 257-267.

(29) Choi, C. H.; Chung, M. W.; Jun, Y. J.; Woo, S. I. RSC Adv. 2013, 3, 12417-12422.

(30) Wang, S.; Zhang, L.; Xia, Z.; Roy, A.; Chang, D. W.; Baek, J. B.; Dai, L. Angew. Chem. Int. Ed. 2012, 51, 4209-4212.

(31) Gong, K.; Du, F.; Xia, Z.; Durstock, M.; Dai, L. Science 2009, 323, 760-764.

(32) Zhang, L.; Xia, Z. J. Phys. Chem. C 2011, 115, 11170-11176.

(33) Liang, J.; Jiao, Y.; Jaroniec, M.; Qiao, S. Z. Angew. Chem. Int. Ed. 2012, 51, 11496-11500. 
(34) Hou, Y.; Chen, Z. P.; Wang, D.; Zhang, B.; Yang, S.; Wang, H. F.; Hu, P.; Zhao, H. J.; Yang, H. G. Small 2014, 10, 484-492.

(35) Hou, Y.; Wang, D.; Yang, X. H.; Fang, W. Q.; Zhang, B.; Wang, H. F.; Lu, G. Z.; Hu, P.; Zhao, H. J.; Yang, H. G. Nat. Commun. 2013, 4, 1583.

(36) Zhang, B.; Wang, D.; Hou, Y.; Yang, S.; Yang, X. H.; Zhong, J. H.; Liu, J.; Wang, H. F.; Hu, P.; Zhao, H. J.; Yang, H. G. Sci. Rep. 2013, 3, 1836.

(37) Zhang, B.; Zhang, N. N.; Chen, J. F.; Hou, Y.; Yang, S.; Guo, J. W.; Yang, X. H.; Zhong, J. H.; Wang, H. F.; Hu, P.; Zhao, H. J.; Yang, H. G. Sci. Rep. 2013, 3, 3109.

(38) Thomas, S.; Deepak, T. G.; Anjusree, G. S.; Arun, T. A.; Nair, S. V.; Nair, A. S. J. Mater. Chem. A 2014, $2,4474-4490$.

(39) Lodermeyer, F.; Costa, R. D.; Casillas, R.; Kohler, F. T. U.; Wasserscheid, P.; Prato, M.; Guldi, D. M. Energ. Environ. Sci. 2015, 8, 241-246.

(40) Lee, K. S.; Lee, H. K.; Wang, D. H.; Park, N.-G.; Lee, J. Y.; Park, O. O.; Park, J. H. Chem. Commun. 2010, 46, 4505-4507.

(41) Tang, Q.; Cai, H.; Yuan, S.; Wang, X. J. Mater. Chem. A 2013, 1, 317-323.

(42) Kresse, G.; Furthmüller, J. Comput. Mater. Sci. 1996, 6, 15-50.

(43) Kresse, G.; Hafner, J. Phys. Rev. B 1994, 49, 14251-14269.

(44) Perdew, J. P.; Burke, K.; Ernzerhof, M. Phys. Rev. Lett. 1996, 77, 3865-3868.

(45) Thonhauser, T.; Cooper, V. R.; Li, S.; Puzder, A.; Hyldgaard, P.; Langreth, D. C. Phys. Rev. B 2007, 76, 125112.

(46) Jiří, K.; David, R. B.; Angelos, M. J. Phys.: Condens. Matter 2010, 22, 022201.

(47) Kresse, G.; Joubert, D. Phys. Rev. B 1999, 59, 1758-1775.

(48) Blöchl, P. E.; Jepsen, O.; Andersen, O. K. Phys. Rev. B 1994, 49, 16223-16233.

(49) Alavi, A.; Hu, P.; Deutsch, T.; Silvestrelli, P. L.; Hutter, J. Phys. Rev. Lett. 1998, 80, 3650-1653.

(50) Liu, Z.-P.; Hu, P. J. Am. Chem, Soc. 2003, 125, 1958-1967.

(51) Cheng, J.; Hu, P. Angew. Chem. Int. Ed. 2011, 50, 7650-7654.

(52) Hauch, A.; Georg, A. Electrochim. Acta 2001, 46, 3457-3466.

(53) Pramanik, A.; Kang, H. S. J. Phys. Chem. C 2011, 115, 10971-10978.

(54) Wu, P.; Du, P.; Zhang, H.; Cai, C. Phys. Chem. Chem. Phys. 2013, 15, 6920-6928.

(55) Yildirim, H.; Kara, A. J. Phys. Chem. C 2013, 117, 2893-2902.

(56) Wang, D.; Jiang, J.; Wang, H. F.; Hu, P. ACS Catal. 2016, 6, 733-741. 
(57) Glenis, S.; Nelson, A. J.; Labes, M. M. J. Appl. Phys. 1999, 86, 4464-4466.

(58) Kheirabadi, N.; Shafiekhani, A.; Fathipour, M. Superlattices Microstruct. 2014, 74, 123-145.

(59) Huang, B.; Yan, Q.-m.; Li, Z.-y.; Duan, W.-h. Front. Phys. China 2009, 4, 269-279.

(60) Magda, G. b. Z.; Jin, X.; Hagymasi, I.; Vancso, P. t.; Osvath, Z. n.; Nemes-Incze, P. t.; Hwang, C.; Biro, L. s. P.; Tapaszto1, L. Nature 2014, 514, 608-611.

(61) Jiao, Y., Zheng, Y., Jaroniec, M. and Qiao, S. Z. J. Am. Chem, Soc. 2014, 136, 4394-4403. 